\title{
A Recurrence Quantification Analysis of Traditional Chinese Medicine Pulse and Its Application in Assessment of Coronary Artery Disease
}

\author{
Rui GUO \\ Institute of Interdisciplinary Research Complex, \\ Key Laboratory of Health Identification and Assessment \\ of Shanghai, \\ Shanghai University of TCM \\ Shanghai, China \\ e-mail: guoruier@sina.com \\ Jianjun YAN* \\ Center for Mechatronics Engineering \\ East China University of Science and Technology \\ Shanghai, China \\ e-mail: jjyan@ecust.edu.cn
}

\author{
Yiqin WANG* \\ School of Basic Medical Science, \\ Key Laboratory of Health Identification and Assessment \\ of Shanghai, \\ Shanghai University of TCM \\ Shanghai, China \\ e-mail: wangyiqing@sina.com
}

\author{
Haixia YAN \\ School of Basic Medical Science, \\ Key Laboratory of Health Identification and Assessment \\ of Shanghai, \\ Shanghai University of TCM \\ Shanghai, China \\ e-mail: hjy2012ok@163.com
}

\begin{abstract}
Purpose: This study was conducted to illustrate that pulse diagnosis of traditional Chinese medicine (TCM) was useful in the assessment of coronary artery lesions in patients with Coronary Heart Disease (CHD). Methods: By using phase-space reconstruction, one-dimensional pulse signals of participants were extended to higher-dimensional phase space, and recurrence plot (RP) and recurrence quantification analysis (RQA) were performed to extract nonlinear dynamic characteristics of pulse signals recorded from the radial artery of participants. Nonparametric test was used to investigate differences of RQA features of the pulse signals between the normal group, the group of single-vessel coronary artery disease and the group of multivessel coronary artery disease. Receiver operating characteristic (ROC) curve analysis was applied to determine the diagnosis value of the RQA features of pulse signals in identifying multivessel coronary artery disease. Results: Among the RQA features of pulse signals, recurrence rate (RR) and trapping time (TT) of the two groups with CHD were significantly greater than those of the normal group $(<0.05)$; $R R$ identified multivessel coronary artery lesions wih an area under ROC curve(AUC) of .891, a maximum

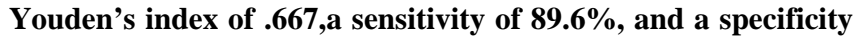
of $\mathbf{8 0 . 2 \%}$; TT identified multivessel coronary coronary artery lesions with an AUC of .812, a maximum Youden's index of 0.573, and a sensitivity of $76 \%$ and a specificity of 81.3\%.Conclusion: RQA features of TCM pulse signals can be used to assess the potential risk of cardiovascular accidents in patients with CHD.
\end{abstract}

Keywords-pulse diagnosis; recurrence quantification analysis; assessment; multivessel coronary artery lesions/single-vessel coronary artery lesion component

\section{INTRODUCTION}

According to 2014 China's cardiovascular disease report [1], cardiovascular disease accounted for more than $40 \%$ of the death of residents, which is the first cause of death for our residents. The burden of cardiovascular disease is increasing, which has become a major public health problem. It is urgent to prevent and cure cardiovascular disease. From 2002 to 2013, the mortality rate of coronary heart disease (CHD) is in the general upward trend, the report said. Whether this disease can be controlled depends on early diagnosis and prevention. In contemporary medicine, methods for detecting CHD include coronary computed tomography (CT), coronary angiography, and cardiovascular ultrasound. Invasiveness or high cost or complicated operation limits using these examination methods to detect $\mathrm{CHD}$ at an early stage. Feasible, noninvasive examination methods are crucial for conserving medical resources and reducing the financial burdens of patients. Previous studies have confirmed that changes in arterial function and structure are the main causes of clinical cardiovascular events[2-4]. Detecting vascular disease at an early stage is extremely crucial for reversing and preventing CHD, stroke, and sudden death. Therefore, developing a noninvasive, convenient, and effective method for detecting vascular disease is essential. The occurrence and development of atherosclerotic disease is a long process. Many studies [5-8] have indicated that cardiovascular parameters change at early stages of cardiovascular disease such as arteriosclerosis; these changes can be identified from changes of pulse signals at the radial artery. Pulse taking of traditional Chinese medicine (TCM) has important clinical value on the diagnosis and prognosis of disease, especially angiocardiopathy. Therefore, visceral disease (particularly, 
changes of vascular structure and function) can be detected through the analysis of pulse signals.

Traditional methods of analysing pulse signals are linear methods (e.g., time-domain and frequency methods). When a traditional method for analysing pulse information is used, nonlinear information is inevitably lost during the process of analysing the pulse information. Numerous studies have shown that changes in physiological signal because of changes in a person's physiological status also demonstrated nonlinear characteristics. An increasing number of researchers have used nonlinear methods to analyse biological signals. A recurrence plot (RP) is an effective tool for investigating the characteristics of nonlinear dynamics, and a nonlinear technique, recurrence quantification analyse (RQA), provides quantitative interpretation of the structures in the RP. In our previous study, RP and RQA were performed to extract nonlinear features of pulse signals. Our previous studies have shown that the RQA features of pulse signals are useful in discriminating between patients with the CHD and the normal people [9-10].

In the present study, we extracted the RQA features of pulse signals in patients with the single-vessel and the multivessel coronary artery disease. And then, the receiver operating characteristic (ROC) curve was drawn to evaluate the diagnostic value of RQA features of pulse signals for distinguishing the multivessel coronary artery disease from the single-vessel coronary artery disease. The purpose of this study was to clarify TCM pulse information can be used to evaluate the potential risk of cardiovascular accidents in patients with coronary heart disease (CHD).

\section{DATA AND METHODS}

TCM pulse refers to what the doctor senses by palpating the examinee's radial artery with fingers. Imitating TCM doctors, a ZM-III intelligent pulse apparatus is employed to acquire pulse recordings at a frequency of $500 \mathrm{~Hz}$, which lays the foundation for objective pulse analysis. The participants, without respiratory system and nervous system disorders, could be supine or seated as their pulses at radial artery (left Cunkou pulses) were measured. Each subject is asked to relax for more than 5 min before pulse acquisition, and their optimal pulse signals were used in the data analysis.

The group with the CHD and the normal group are studied:

- The group with the CHD includes 144 inpatients aged $63.37 \pm 8.74$ years, collected by our research team at the inpatient wards of Shanghai Municipal Hospital of Traditional Chinese Medicine, and Shanghai Shuguang Hospital from October 2006 to October 2007. Among the 144 patients in this study, 96 patients experienced single-vessel coronary artery disease and 48 patients experienced multivessel coronary artery disease according to the coronary angiography report.

- The normal group includes 69 normal subjects who are selected as control subjects aged $55.26 \pm 10.36$ years from employees at the Shanghai University of Traditional Chinese Medicine. In the control group, none had a history of organic disease or cardiovascular abnormalities, and all had normal blood pressure.

\section{MEthod FOR ANALysing PUlse SignAls}

\section{A. Recurrence Quantification Analysis}

The nonlinear method was employed to analyse pulse signal here. For nonlinear analysis, phase space reconstruction is basis of analyzing complex system, and its basic idea is that the evolution of any component of a system is related to the other components of the system, and thus the information of these components is necessarily implicit in the evolution of the other component. So we only need to measure one of those components to learn the underlying dynamics characteristics of the system. It have been demonstrated that a single measured component (onedimension signal or time series data) of the system can reconstruct (be embedded into) higher-dimensional phase space of that system by the method of time delays [11]. Thus we can learn about the dynamics characteristics of system in higher-dimensional space, not obvious in the one-dimension signal. It must be appreciated that the selections of embedding dimension and delay are based on nonlinear dynamical theory. Embedding dimension can in principle be estimated by the nearest-neighbour methodology [12]. Proper delay can be selected to find the first minimum in mutual information function of the continuous time series [13]. For example, the one-dimension pulse signal (one minute of time series data) recorded from the radial artery of a CHD patient was showed in Fig 1 and its attendant reconstructed phase space with topological features equivalent to actual pulse system was showed in Fig. 2.

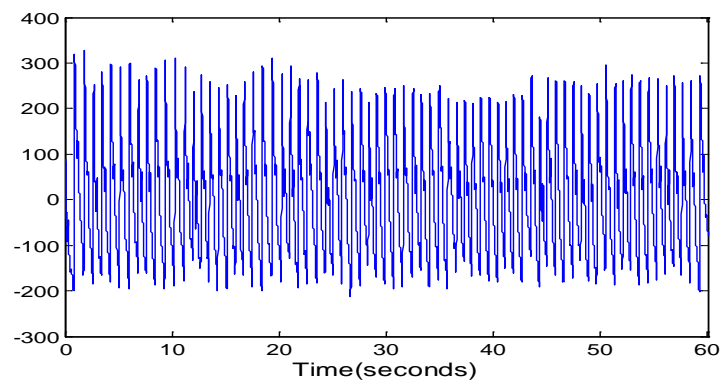

Figure 1. Time series of a one-dimension, a pulse signal recorded from a CHD patient for one minute.

Fig. 2 showed the phase-space trajectory of pulse system. To reproduce the behaviour of phase-space trajectory dynamics, a RP was constructed shown in Fig. 3 for investigating the characteristics of nonlinear dynamics. A RP [14] is an effective tool to visualise recurrences of higherdimensional phase space trajectories. The RP consists of a two-dimensional squared matrix with black and white dots, where black dots marked a recurrence and both axes were time axes. RP's qualitative examination revealed line segments parallel to the central diagonal, a cluster of recurrence points, and a few isolated points representing chance recurrences. Isolated points occurred on the RP because trajectories instantaneously approached each other 
and rapidly separated, indicating an unstable system evolution. Vertical and horizontal line segments had identical dynamic implications, signifying that a relatively slow change occurred at a certain time point. The length of the line segment running parallel to the main diagonal line reflected the velocity with which adjacent phase-space trajectories separated from each other. For example, the most striking feature of the plot in Fig. 3 was the diagonal line structures parallel to the main diagonal.

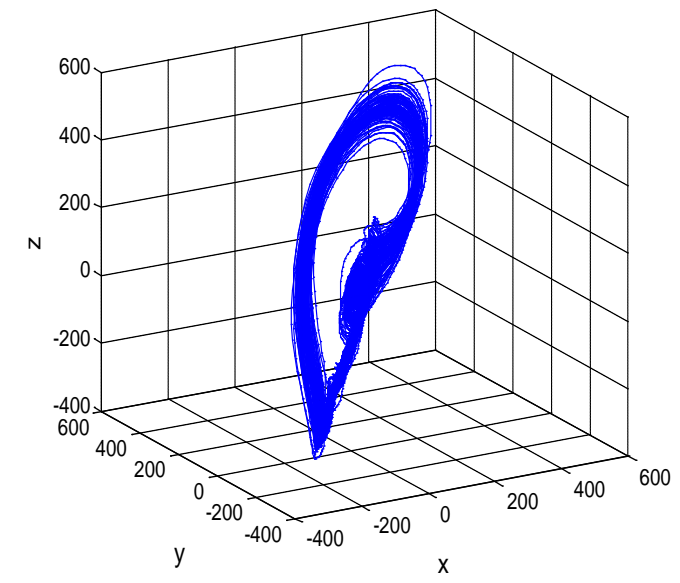

Figure 2. Three-dimensional reconstruction of the pulse signal in phase space by the method of time delays (delay=4 points)
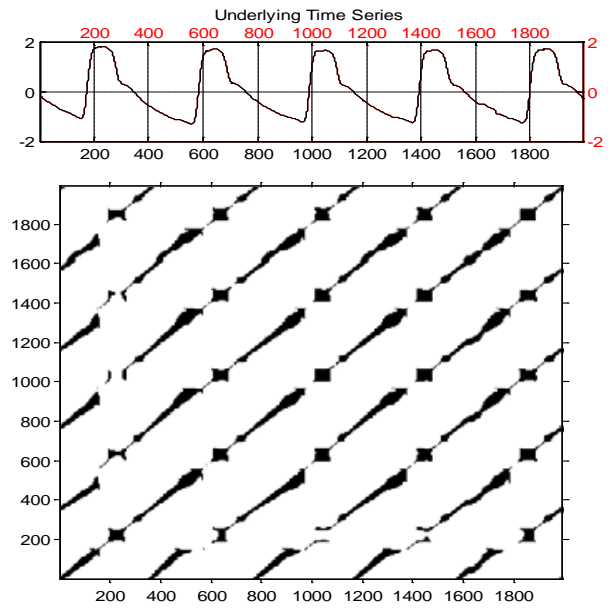

Figure 3. Recurrent plot of the pulse signal with window size of 2000 pints

A RP was a visualisation tool, what yielded to the advantage that the user had to detect and interpret the patterns and structures revealed by the PR. To overcome the subjective interpretations of RP, Zbilut and Weber [15-16] introduced the known measures of complexity based on recurrence dot and diagonal line structures and therewith established RQA. Seven RQA features (recurrence variables) are computed (extracted) from the RP within each window of observation on the time series. By implementing a sliding window design, each of those features is computed multiple times, creating seven new derivative dynamical system expressed in terms of recurrence rate (RR), determinism (DET), linemax (LMAX), entropy (ENT), trend (TND), laminarity (LAM), trapping time (TT). These features (outputs) with the original time series (input) might reveal different dynamic characteristics in a system, not obvious in the one-dimensional input data.

In this experiment, we focused on the two of RQA features: RR and TT. The equations for calculating RR and TT were as follows:

$$
R R=\frac{1}{N^{2}} \sum_{i, j}^{N} R_{i, j}
$$

where RR quantifies percentage recurrences or recurrence rate. This variable can range from $0 \%$ (no recurrent points) to $100 \%$ (all points recurrent). Large RR implies a strong periodic embedding process in the system. Thus, the bigger the $\mathrm{RR}$, the more regular the signal is.

$$
T T=\frac{\sum_{v=v_{\min }}^{N} v P(v)}{\sum_{v=v_{\min }}^{N} p(v)}
$$

where TT is simply the average length of vertical line structures, measuring the average duration that a system in a specific state. Thus, the bigger the TT, the more stable the signal is.

\section{B. Statistical Methods}

SPSS Version 20 software was used for statistical analysis in this study. By observing the distribution of the RQA features (RR and TT) of the pulse signals of patients with CHD (single-vessel and multivessel coronary artery disease) and those of normal people, we determined that the statistical variables were not normally distributed and did not meet the assumptions of homogeneous variances. Therefore, nonparametric test was used to investigate differences in the ROA features of the three groups' pulse signals. Regarding descriptive statistics, median and inter-quartile range (IQR) were used to describe the degree of dispersion (i.e., M (QLQU)).

\section{Statistical Methods}

In statistics, ROC curve is a graphical plot that illustrates the performance of a binary classifier system as its discrimination threshold or critical value is varied, which have been widely applied in assessing the effectiveness of medical diagnosis. In an ROC graph, the curve is generated by calculating the sensitivity (true positive rate) against the (1-specificity) (false positive rate) at various critical values, $\mathrm{x}$-axis and $\mathrm{y}$-axis represent (1-specificity) and the sensitivity respectively. Each prediction result (the sensitivity and specificity) represents one point in the ROC curve. The closer a result (point in the curve) is to the upper left corner, the better it predicts. 
The area under the ROC curve (AUC) represents the degree of overlap between positive and negative diagnostic results in a diagnosis system, and the larger the AUC, the higher the diagnostic value of a model or predictor. Researchers in the medical field consider AUC of between .5 and .7 to be a low corresponding diagnostic value, AUC of between .7 and .9 to be a medium corresponding diagnostic value, and AUC greater than .9 to be a high corresponding diagnostic value [17].

\section{RESULTS}

\section{A. RQA of Pulse Signals of The Participants}

Because the RQA features of the pulse signals in the three groups were not normally distributed and did not meet the assumptions of homogeneous variances, nonparametric test was used to investigate differences in the ROA features of the three groups' pulse signals. Table I showed the result of the nonparametric test on the ROA features of the three groups' pulse signals after correcting age effect on statistical model. The RQA features of the pulse signals of the group with CHD were significantly greater than those of the normal group; the RQA features of the pulse signals of the patients with multivessel coronary artery disease were significantly greater than those of the patients with single-vessel coronary artery disease.

TABLE I. RQA FEATURES OF PULSE Signals of THE THREE GRoups (M(QL-QU))

\begin{tabular}{clcc}
\hline Group & Num & RR & TT \\
\hline the normal & 69 & $0.057(0.052-0.064)$ & $10.786(9.293-12.005)$ \\
the single-vessel & 96 & $0.067(0.06-0.074)^{*}$ & $13.353(11.525-14.870)^{*}$ \\
the multivessel & 48 & $0.088(0.080-0.995) * \boldsymbol{\Delta}$ & $16.782(15.227-20.059)^{*} \boldsymbol{\Delta}$ \\
\hline
\end{tabular}

Note: Comparison with the normal group: ${ }^{*} \mathrm{p}<.05$; comparison with the group with single-vessel coronary artery disease: $\boldsymbol{\Delta} \mathrm{p}<.05$

\section{B. Determining The Diagnostic Value for Multivessel Coronary Artery Disease}

1) ROC cure of RR and TT:In the ROC curve analysis, the predicted objects refer to the positive or negative result of the test, the general said to be mainly two classified variables. In this study, the ROC curve was used to determine the diagnostic values of RR and TT for identifying multivessel coronary artery disease (shown in Fig. 4 and Table II), the group with single-vessel coronary artery disease served as the control group. As shown in Table II, AUC of RR and TT were .891and .812, respectively. The two values were both greater than .7 , indicating satisfactory diagnostic value.

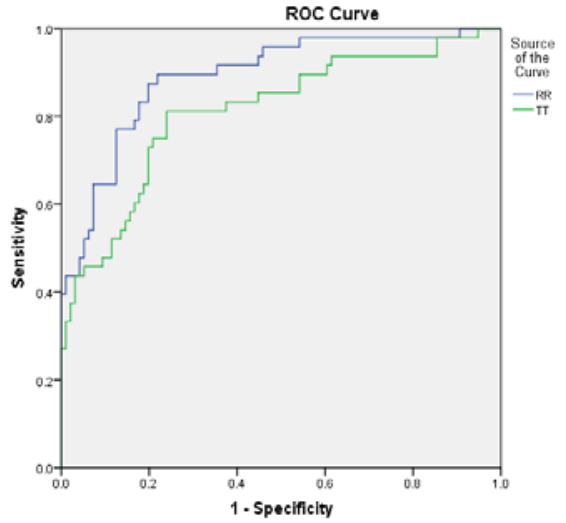

Figure 4. ROC curve of RR and TT as predictors for diagnosing multivessel coronary artery disease

TABLE II. AUC OF RR AND TT

\begin{tabular}{cccc}
\hline Test Variables & AUC & Std.Error & P \\
\hline RR & 0.891 & 0.029 & $0.000<0.01$ \\
TT & 0.812 & 0.040 & $0.000<0.01$ \\
\hline
\end{tabular}

2) Determining the diagnostic point: A ROC curve is typically plotted with critical values representing the sensitivity and specificity. Therefore, an optimal critical value (diagnostic point) must be selected to determine a diagnosis criterion. Optimal results can be obtained when both the maximum sensitivity and specificity are reached at a certain critical value. However, a common phenomenon is that the maximum sensitivity occurs at one critical value while the maximum specificity occurs at another critical value. Some studies have considered that screening tests emphasize the importance of high sensitivity but that diagnostic tests emphasize the importance of high specificity; thus, a critical value can be set according to clinical requirements. Disregarding the aforementioned factors, we selected the Youden's index close to the upper left part (maximum Yonden's index) of the ROC curve as the optimal critical value. The Youden's index equals the results of subtracting 1 from the sum of sensitivity and specificity, indicating the total capacity of a selection method for distinguishing between patients and nonpatients. A larger Youden's index indicates excellent and valid screening results.

Table III showed the diagnostic points (the optimal clinical value), the maximum Youden's index, as well as the sensitivity and specificity when RR and TT were used to diagnose multivessel coronary artery disease. When the diagnostic points of RR is 0.076 at the maximum Youden index of 0.667 , the sensitivity and the sensitivity are 0.896 and 0.781 respectively, when the diagnostic points of TT is 14.934 at the maximum Youden index of 0.572 , both the referred valued are 0.760 and 0.813 . 


\section{DISCUSSION}

In this study, RP and RQA technique were employed to extract the nonlinear dynamics features of pulse signals in the groups of the healthy people and of patients with CHD. Various RQA features describe different dynamic implication in a system. RR measures the recurrence frequency of state points and their aggregation in the phase space. The bigger the RR, the more regular the system is. TT measures the average length of vertical or horizontal line segments, representing the response speed of a system (i.e., system stability). The bigger the TT, the more stable the system is. From our results, we determined that the RR and TT of the two groups with CHD were significantly greater than those of the normally group. These results agree with our previous studies [5, 6]. Moreover, the RR and TT of the patients with multivessel coronary artery disease were significantly greater than those of the patients with singlevessel coronary artery disease. Namely, the RPs of patients with multivessel coronary artery disease contained more recurrence points, indicating an apparent periodic embedding process. According to the implication of $\mathrm{RR}$ in nonlinear dynamics, we inferred that the pulse signals of the patients with multivessel coronary artery disease were higher regular than the other groups. Likewise, the RPs of patients with multivessel coronary artery disease contained the longer length of vertical or horizontal line segments, indicating a slow change in the phase-space trajectory of the pulse signals of patients with multivessel coronary artery disease, which suggested that the pulse signals of the patients with multivessel coronary artery disease were higher stable than the other groups. These results were consistent with that of previous studies reported; i.e., which physiological systems are stable and regular (low complexity) under pathological conditions. Researchers typically consider that a reduction in the complexity of a system frequently accompanies pathological changes, and the complexity reduction has been pointed out to be a common feature in the pathological state. The nonlinear dynamic characteristics of pulse provide a better method for characterizing pathological state.

Early discovery of coronary artery lesions is important for the prevention of cardiovascular events. In this study we found that RR and TT of RQA features of pulse signals can be used to identify multivessel coronary artery disease, because of respectively yielding AUC of .891 and .812. It can be seen from Table III that, when the RR was greater than .076, we predicted that the patient with CHD experienced multivessel coronary artery disease with the sensitivity of $89.6 \%$ and the specificity of $78.1 \%$, when TT was greater than 14.934, we predicted that with the sensitivity of $76 \%$ and the specificity of $81.3 \%$. RR had a higher sensitivity of $89.5 \%$ for identifying multivessel coronary artery disease; however, TT had a higher specificity of $81.3 \%$ for that. Therefore, when clinical trials require a higher sensitivity, RR maybe be chosen as a predictor of multivessel coronary artery disease; when if require a higher specificity, TT maybe be chosen for that. Also, according to the clinical practice to determine the required sensitivity and specificity, linear interpolation can be used to find out the diagnostic values of RR and TT.

The study found that the pulse information can be used to estimate severity of coronary atherosclerosis, helping to assess the potential risks and development of cardiovascular disease in patients with CHD, and to remind clinicians to give effective intervention early to reduce the occurrence of cardiovascular accidents. Pulse diagnosis technology as a noninvasive and convenient screening technique for arteriosclerosis will become a powerful complement to the existing technology.

\section{ACKNOWLEDGMENT}

This work was supported by National Natural Science Foundation of China for Youth under Grant No. 81302913, Innovation Program of Shanghai Municipal Education Commission under Grant No.11YZ71, and National Natural Science Foundation of China under Grant No. 81173199 \& No. 81473594. Key Laboratory of Health Identification and Assessment of Shanghai, Shanghai University of TCM TCM Diagnosis Leading Academic Discipline Project of State Administration of TCM of China, Special Fund of Technical Standards by Science and Technology Commission of Shanghai (14DZ0500400) Thanks are due to all of volunteers for providing invaluable pulse data.

\section{REFERENCES}

[1] Chen Weiwei, Gao Runlin, Liu Lisheng,et al. Outline of "China cardiovascular disease report 2014”. Chinese Circulation Journal, 2015, 30(7): 617-622.

[2] WANG Hong-yu, WANG Jing, LIU Wang-peng, et al.. Application of early vascular disease detection system in patients with cardiovascular disease.Medical Journal of Chinese People's Health, 2006,18(5):336-339

[3] Ito Mk, Talbert RL, Tsimikas S. Stain-Associated Pleiotropy: Possible Beneficial Effect Beyond Cholesterol Reduction [J]. Pharmacotherapy: The Journal of Human Pharmacology and Drug Therapy 2006, 26(7P2): 855-975

[4] Wang Xueyan, Mao Song. The comparative observation of the cariotid intima-media thickness and endothelial function of patients of high blood pressure complicated coronary heart disease and stroke[J]. Modern Medicine Journal of China,2010,12(1): 42-44

[5] Rui Guo, Yiqin Wang, Haixia Yan. Study on TCM pulse of the patient with coronary heart disease based on recurrence quantification analysis. J Chin Integr Med. 2011, 9(11): 1226-1233

[6] GUO Rui, WANG Yiqin, YAN Haixia, et al. A Recurrence Quantification Analysis of Traditional Chinese Medicine Pulse and a Pulse-based Assessment of Carotid Atherosclerosis of the Patients with Coronary Heart Disease. China Journal of Traditional Chinese Medicine and Pharmacy,2016,31(5): 1695-1699.

[7] Kang Ping, Li Fufeng, Yao Di, et al. Association Analysis on Pulse Graph Characters of Coronary Heart Disease and Its Blood-fat and Thrombin. World Science and Technology/Modernizing of Traditional Chinese Medicine and Materia Medica, 2011, 13(5): 810815.

[8] He Wei, Yu Chuanxiang. Principle and clinical application of cardiovascular dynamics parameters measurement[M]. Science and Technology Publishing Press, Beijing, 2010

[9] Rui Guo, Yiqin Wang, Haixia Yan,et al.Recurrence quantification analysis on pulse morphological changes in patient with coronary heart disease. Journal of Traditional Chinese Medicine, 2012, 32(4):571-577

[10] Rui Guo, Yiqin Wang, Jin Xu, Haixia Yan, Jianjun Yan, Fufeng Li, Zhaoxia Xu and Wenjie Xu. Research on Zheng Classification Fusing 
Pulse Parameters in Coronary Heart Disease, Evidence-Based Complementary and Alternative Medicine, vol. 2013, Article ID 602672, 8 pages, 2013. doi:10.1155/2013/602672

[11] Ryan TJ, Faxon DP, Gunnar RM, et al. A report of ACC/AHA task force on assessment of diagnostic and therapeutic cardiovascular procedures. J Am CollCardiol, 1988, 12(3): 529.

[12] Takens F. Detecting strange attractors in turbulence. Lecture Notes in Mathematics. 1981, 898(1): 366-381.

[13] kennel, M.B., Brown, R. and Abarbanel, H.D.I..Determining Minimum Embedding Dimension Using a Geometrical Construction. Physical,Review A,1992,45,3045-3411
[14] Frazer, A.M., Swinney, H.L..Independent coordinates in strange attractors from mutual information. Physical. Review A, 1986,33,1134-1140

[15] Eckmann J.P., Kamphorst S.O., Ruelle D., Recurrence Plots of Dynamical System Europhysics. Letter.1987, 4: 973-977

[16] Zbilut JP, Webber CL. Embeddings and delays as derived from quantification of recurrence plots. Physics Letters A. 1992, 171: 190203

[17] Webber CL, Zbilut, JP. Dynamical assessment of physiological systems and states using recurrence plot strategies. Journal of applied Physiology. 1994, 76(2): 965-973

[18] Swets JA. Measuring the accuracy of diagnostic systems. Science 1988, 240: 1285-1293

TABLE III. Diagnostic VALUe of RR AND TT FOR INDENTIFying MULTIVESSEL CORONARY ARTERy DiseASE

\begin{tabular}{cccccc}
\hline Test Variables & Maximum Youden index & Diagnostic value & AUC & Sensitivity & Specificity \\
\hline RR & 0.667 & 0.076 & 0.891 & 0.896 & 0.781 \\
TT & 0.573 & 14.934 & 0.812 & 0.760 & 0.813 \\
\hline
\end{tabular}

